\title{
Compact radio components in Seyfert nuclei
}

\author{
D.Taylor, J.E.Dyson, A.Pedlar, D.J.Axon, (University of Manchester, Manch- \\ ester,M19 9PL, UK.) \& S.W.Unger (Royal Greenwich Observatory, Sussex, UK.)
}

Although initial, low resolution radio measurements by deBruyn and Wilson (1978), suggested that the radio emission in Seyfert nuclei was in approximate pressure equilibrium with thermal gas in the Narrow Line Region (NLR), higher resolution radio measurements using the VLA and MERLIN (eg Unger et al. 1985) showed that the radio components were at significantly higher pressures than the NLR thermal gas. This is even more apparent in the very long baseline interferometry (VLBI) observations using the European Network (EVN). Observations of NGC4151 (Harrison et al.1986) and NGC7674 (Unger et al. 1988) have already been described, together with a summary of work in progress by Pedlar et al. 1987 . Several Seyfert nuclei have components in which relativistic particle/magnetic field pressures as high as $10^{-7}$ dynes $/ \mathrm{cm}^{2}$ are infered. These observations are also consistent with the collimated ejection of either radio emitting 'plasmons' or beams from the optical nucleus.

Given that in the static case these radio components are at considerably higher pressures than their surroundings we have considered two possible models: one in which the radio component is at rest with respect to the ambient medium and expands radially driving shocks into its surroundings ( Pedlar,Dyson \& Unger 1985), and a second in which the radio components are moving through the medium and are confined by ram pressure (Pedlar et al. 1986). Optical Observations of the NLR in NGC5929 (Whittle et al. 1986), Mkn78 (Pedlar et al. 1988 in prep.), and NGC1068 (Meaburn \& Pedlar 1986) have shown individual [OIII] components to be closely associated with radio emission, a natural consequence of these types of model. The second model has been the subject of a detailed theoretical study by Taylor (1988 in prep.) in which the consequences of an axisymmetric bowshock driven by a plasmon moving at constant velocity $V_{s}$ through an homogenous medium of density $n_{a}$ is investigated. The ambient gas is assumed to be completely photoionised by UV radiation from the nucleus. This gas is swept up and heated by the bowshock, and flows, without turbulence along the bowshock surface. Individual parcels of the post shock gas are assumed to be thermally isolated from their surroundings and cool independantly both adiabatically and radiatively. The ionisation states of the gas elements are estimated by assuming ionisation equilibrium. At any point on the bowshock surface each gas parcel is assumed to have a pressure equal to the ram pressure at that point, and a velocity determined by global conservation of mass and momentum. Hence, at a given distance behind the apex of the bowshock the gas consists of individual pack- 
ets each with a their unique physical structure, but with the same pressure and velocity. The [OIII] emission from each point is calculated by summing the contributions from these elements. The [OIII] flux and line profiles can be constructed by summing contributions from each element.

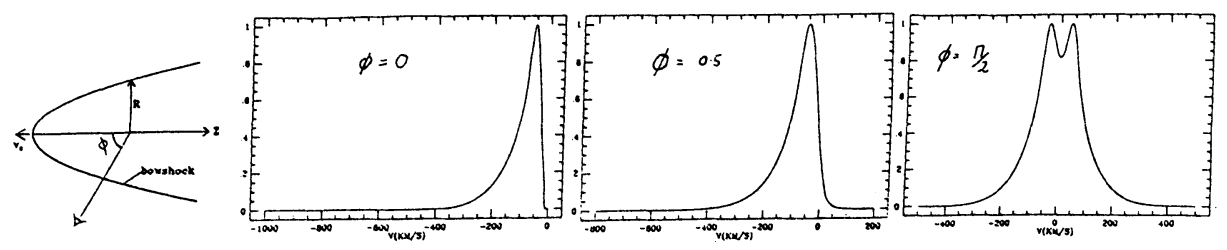

Figure 1 shows an example of theoretical line profiles obtained from a MillsSturrock (1970) plasmon moving with a velocity of $1000 \mathrm{~km} / \mathrm{s}$ in an ambient medium of density 1 atom $\mathrm{cm}^{-3}$. The three profiles represent emission as seen from three different viewing angles $\phi$, which turns out to be the most important single parameter in determining the observed line profiles. The plasmon shape and velocity, and the density of the ambient medium, have considerably less effect than $\phi$. In principle, this property can be used to constrain the geometry of the ejection, and will enable us to determine the true angle of ejection of such plasmons in relation to the rotation axis of the galaxy.

Modelling is currently in progress to fit the line profiles and relative intensities of [OIII] components within a number of Seyfert Nuclei. In particular the line profiles of the two [OIII] components in NGC5929 shown in Whittle et al. (1986) can be closely reproduced by two plasmons moving 30 degrees to the line of sight with velocities of 650 and $1000 \mathrm{~km} / \mathrm{s}$ respectively (Taylor et al. 1988 in prep.). The observed velocity difference between the two components can be reproduced either by rotation or radial outflow of the ambient medium, although mechanisms by which a higher fraction of the plasmon velocity can be transferred to the [OIII] are currently under investigation.

\section{References}

deBruyn,A.G. \& Wilson,A.S.,1978, Astr. Astrophys,64,433.

Harrison et al.,1986,MNRAS,218,775.

Meaburn, J. \& Pedlar,A.,1986,Astron Astrophys.,159, 336.

Mills,D.M. \& Sturrock, P.A.,1970, Astrophys Letters,5,105.

Pedlar, A., Dyson, J.E. \& Unger S.W., 1985, MNRAS, 214, 463.

Pedlar, A. et al.,1986. Star formation in Galaxies, NASA Conf. Publ. 2466, p 711.

Pedlar et al., 1987, " Active Galactic Nuclei", Atlanta, October 1987.

Unger et al. 1985, MNRAS.,219,387.

Unger et al. 1988, MNRAS, (in press)

Whittle et al.,1986,MNRAS,222,189. 